\title{
Akt enhances nerve growth factor-induced axon growth via activating the Nrf2/ARE pathway
}

\author{
BIN XIA ${ }^{1}$, HEYU LIU ${ }^{1}$, JUANKE XIE ${ }^{2}$, RUI WU $^{1}$ and YALI LI ${ }^{1}$ \\ ${ }^{1}$ Department of Neurological Rehabilitation, The Second Affiliated Hospital of Zhengzhou University, Zhengzhou, \\ Henan 450014; ${ }^{2}$ Reproductive Medicine Center, Henan Provincial People's Hospital, Zhengzhou, Henan 450003, P.R. China
}

Received January 30, 2015; Accepted August 20, 2015

DOI: $10.3892 /$ ijmm.2015.2329

\begin{abstract}
Spinal cord injury (SCI) leads to the loss of structure and function of axons. However, injured axons cannot grow or regenerate spontaneously following injury. Generally, only when treated with neurotrophins, such as nerve growth factor (NGF), will the neurons sprout new axons. Akt is one of the central kinases of neurocytes. PC12 cells are a frequently used cell model for neural differentiation and development studies. The nuclear factor erythroid 2-related factor 2 (Nrf2)/antioxidant response element (ARE) signaling pathway is a main mechanism in prevention from oxidative stress, which may damage the nervous system. The present study employed this cell model to investigate whether Akt could induce axon growth in PC12 cells on the basis of NGF treatments. The results showed that Akt overexpression significantly increased cell proliferation and decreased cell apoptosis. Additionally, Akt overexpression activated Nrf2/ARE pathways. In conclusion, the experiments indicated that Akt overexpression contributed to axon regeneration induced by NGF in PC12 cells through activating the Nrf2/ARE pathway.
\end{abstract}

\section{Introduction}

Spinal cord injury (SCI) leads to the consequent death of neurocytes, thus causing the dysfunction of signal transmissions between neurons and axons, which could in turn induce apoptosis of neurons. As reported, axons of the injured neurons in the adult mammalian central nervous system (CNS) can

Correspondence to: Dr Bin Xia, Department of Neurological Rehabilitation, The Second Affiliated Hospital of Zhengzhou University, 2 Jingba Road, Zhengzhou, Henan 450014, P.R. China E-mail: binxiasec@163.com

Abbreviations: SCI, spinal cord injury; NGF, nerve growth factor; Nrf2, nuclear factor erythroid 2-related factor 2; ARE, antioxidant response element; CNS, central nervous system; HO-1, heme oxygenase-1; NQO-1, NAD(P)H:quinone oxidoreductase-1; $\gamma$-GCS, $\gamma$-glutamylcysteine synthetase

Key words: spinal cord injury, axon growth, Akt, nerve growth factor, PC12 cells seldom spontaneously regenerate (1). This phenomenon has caused confusion for the SCI treatment. There are relevant studies that have restored the regenerative ability of the injured axons (2-11). For example, various neurotrophin treatments, including nerve growth factor (NGF) and brain-derived neurotrophic factor, have been effectively proved to promote neurons branching and sprouting $(12,13)$, and to promote axon regeneration $(14,15)$. However, the mechanism underlying neurotrophin treatments has not been fully investigated or established.

PC12 cells originate from a rat pheochromocytoma tumor cell line, which is sensitive to NGF treatment by differentiating into neuron-like cells $(16,17)$. Therefore, PC12 cells have been employed as a promising, unique and frequently used cell model for neural development and protection studies $(17,18)$. Akt is a type of neurocyte protein kinase that is associated with stress response to growth factors (19), which also has a role in tumor growth (20). The nuclear factor E2-related factor 2 (Nrf2) is a type of transcription factor, which could initiate antioxidant response element (ARE) transcription. The Nrf2 gene products include a scope of antioxidative factors participating in antioxidant function, such as heme oxygenase-1 (HO-1), NAD(P) H:quinone oxidoreductase-1 (NQO-1) and $\gamma$-glutamylcysteine synthetase ( $\gamma$-GCS) (21-24). The present study aimed to further understand whether Akt could activate Nrf2/ARE antioxidant systems to decrease apoptosis of neurocytes and contribute to axon regeneration.

The NGF-differentiated PC12 cells were used to investigate the effect of Akt on axon growth. Changes in axon regrowth produced by silence and overexpression of Akt were examined and the function of antioxidant enzyme activities in the presence of NGF in PC12 cells was identified. Understanding the mechanisms between Akt and the Nrf2/ARE pathway in PC12 cells is important for the development of new methods to prevent or treat neurodegenerative diseases, such as SCI-caused axon degeneration.

\section{Materials and methods}

Cell culture and differentiation. PC12 cells were purchased from Riken Cell Bank (Tsukuba, Ibaraki, Japan) and cultured in Dulbecco's modified Eagle's medium (DMEM) supplemented with $10 \%(\mathrm{v} / \mathrm{v})$ horse serum and $5 \%(\mathrm{v} / \mathrm{v})$ fetal bovine serum (FBS) (all from Hyclone, Logan, UT, USA). The dishes had 
been previously coated with poly-L-lysine (Sigma-Aldrich, St. Louis, MO, USA). The cells were incubated at $37^{\circ} \mathrm{C}$ in a humidified $5 \% \mathrm{CO}_{2}$ atmosphere. $\mathrm{PC} 12$ cells were differentiated with $100 \mathrm{ng} / \mathrm{ml}$ NGF (Invitrogen, Carlsbad, CA, USA) for $\leq 72 \mathrm{~h}$.

Recombinant adenovirus construction and transfection. Recombinant adenovirus vectors were purchased from Genomeditech Biotechnologies (Shanghai, China). Briefly, the genes encoding Akt were amplified and identified, followed by conjugation with shuttle vector pAdTrack-CMV. The pAdTrack-CMV and adenoviral gene expression vector pAdEasy-1 were co-transfected into HEK293 cells in non-serum DMEM medium to produce recombinant adenovirus using Lipofectamine 2000 (Invitrogen). The recombinant adenoviruses were harvested, amplified, concentrated and purified, and the titers were measured prior to use. Cells treated with empty carrier LacZ instead of recombinant adenovirus were used as negative control.

Preparation of small interference RNA (siRNA) and transfection. The siRNA was synthesized by GenePharma Co., Ltd. (Shanghai, China). Briefly, the medium had been changed to non-serum medium $30 \mathrm{~min}$ before transfection. siRNA (5 $\mu \mathrm{l}$; Akt siRNA sc-108059; Santa Cruz Biotechnology, Inc., Dallas, TX, USA) was added into $245 \mu \mathrm{l}$ of non-serum DMEM (solution A). Lipofectamine 2000 (10 $\mu \mathrm{l}$; Invitrogen) was also diluted in $245 \mu \mathrm{l}$ of non-serum DMEM (solution B) and incubated for $5 \mathrm{~min}$ at room temperature. Subsequently, solution B was gently added into solution A, mixed and incubated for $20 \mathrm{~min}$ at room temperature. The mixtures were equally distributed into the 6-well cultured cells at drop speed with successive agitation, followed by incubation at $37^{\circ} \mathrm{C}$, prior to conducting further analysis. Cells treated with siRNA instead of Akt siRNA were used as the negative control.

Neurite outgrowth measurement of PC12 cells. The length of axons that extended from cell bodies was measured by Image-Pro Plus 6.0 software (Media Cybernetics, Silver Spring, MD, USA). Subsequently, the number of neurites extending from cells was also calculated by counting the number and percentage to determine differentiation efficiency.

3-(4,5-Dimethylthiazol-2-yl)-2,5-diphenyltetrazolium bromide (MTT) assay for cell viability evaluation. Cell viability was evaluated by the MTT assay. Briefly, PC12 cells were cultured in 96-well plates with a density of $2 \times 10^{4}$ cells/well. Subsequently, cells were incubated with $20 \mu \mathrm{l}$ MTT solution $(5 \mathrm{mg} / \mathrm{ml})$ in fresh medium (10\% FBS) for $4 \mathrm{~h}$ in a $37^{\circ} \mathrm{C}$ incubator. Following this, the mixtures were centrifuged at $12,890 \mathrm{x}$ g for $15 \mathrm{~min}$ and the supernatant was carefully discarded using a vacuum pump, and formazan crystals were dissolved in dimethylsulfoxide (0.1\% final concentration; Sigma-Aldrich). The absorbance of samples was measured at $490 \mathrm{~nm}$ using the EnVision ${ }^{\circledR}$ Multilabel Reader (Perkin-Elmer, Waltham, MA, USA).

Hoechst assay and terminal deoxynucleotidyltransferase-mediated dUTP nick end labeling (TUNEL) experiment for apoptosis evaluation. For the Hoechst assay, cells were seeded at a density of $1 \times 10^{4} /$ well in 96 -well plates, followed by the addition of $200 \mu \mathrm{l}$ fresh medium and incubation at $37^{\circ} \mathrm{C}$ in
$5 \% \mathrm{CO}_{2}$. When cells grew to a confluence of $80 \%$, apoptosis was detected via the Hoechst Staining kit (Beyotime, Beijing, China) according to the manufacturer's instructions. For the TUNEL experiment, cells were firstly fixed with $4 \%$ paraformaldehyde for $10 \mathrm{~min}$ at room temperature. Subsequently, cells were washed with phosphate-buffered saline (PBS) twice and permeabilized by $0.1 \%$ Triton X-100 under ice-cold incubation for $10 \mathrm{~min}$. Following washing with PBS again, cell apoptosis was evaluated by a TUNEL Apoptosis Detection kit (Merck Millipore, Billerica, MA, USA) following the manufacturer's instructions. Cells were observed under a fluorescent microscope (Olympus, Tokyo, Japan). The positive cells were counted in randomly selected fields.

Reverse transcription-quantitative polymerase chain reaction $(R T-q P C R)$. Total RNA of targeted PC12 cells was isolated using the TRIzol reagent (Life Technologies, Rockville, MD, USA). Reverse transcription was conducted using $1 \mu \mathrm{g}$ of total RNA from each sample via the oligo(dT) primer, using the RevertAid First Strand cDNA Synthesis kit (Thermo Fisher Scientific, Waltham, MA, USA). RT-qPCR analysis, including Akt, HO-1, NQO-1 and $\gamma$-GCS, was performed using the SYBR-Green PCR kit (Takara, Shiga, Japan) on a Bio-Rad CFX96 Real-Time PCR Detection system. $\beta$-actin served as the reference gene and data were further analyzed using the $\Delta \mathrm{Ct}$ method.

Western blot analysis. Proteins were harvested using radioimmunoprecipitation assay buffer supplemented with protease inhibitor phenylmethanesulfonylfluoride (both from Sigma-Aldrich). A total of $20 \mu \mathrm{g}$ proteins were fractionated via sodium dodecyl sulfate-polyacrylamide gel electrophoresis to be transferred onto a nitrocellulose (NC) membrane (Amersham, Little Chalfont, UK). Subsequently, the NC membrane was incubated in blocking buffer consisting of $4 \%$ bovine serum albumin (Sigma-Aldrich) in Tris-buffered saline to block non-specific binding for $1 \mathrm{~h}$. Subsequently, the membrane was incubated with primary antibodies (rabbit antibodies including Akt (sc-8312; 1:500), HO-1 (sc-10789; 1:500), NQO-1 (sc-16464; 1:600) and $\gamma$-GCS (sc-22755; 1:500), all from Santa Cruz Biotechnology, Inc.) diluted in blocking buffer overnight at $4^{\circ} \mathrm{C}$. On the following day, the $\mathrm{NC}$ membrane was incubated with horseradish peroxidase-conjugated goat anti-rabbit secondary antibody (sc-2004; diluted at 1:1,000; Santa Cruz Biotechnology, Inc.) for $1 \mathrm{~h}$. The protein signal was visualized using the Amersham ECL ${ }^{\text {TM }}$ Plus Western Blotting Detection kit (GE Healthcare, Piscataway, NJ, USA).

Statistical analysis. All the data are presented as mean \pm standard deviation. Comparisons between the two groups and among multiple groups were performed by Student's t-test and one-way analysis of variance, respectively. $\mathrm{P}<0.05$ was considered to indicate a statistically significant difference. All the statistical analyses were performed using SPSS version 19.0 (IBM Corp., Armonk, NY, USA).

\section{Results}

NGF promotes neural differentiation of PC12 cells. The study first confirmed the function of NGF to induce neural differentiation of PC12 cells. NGF-treated cells had a significant 

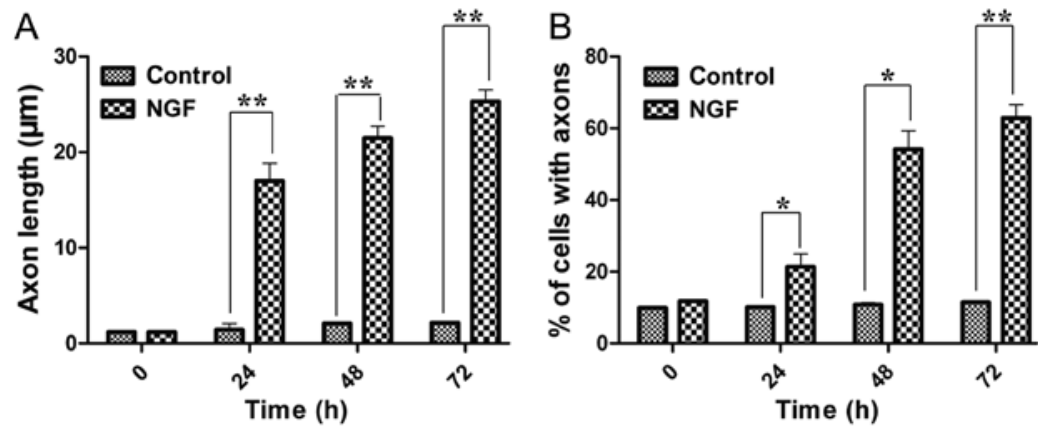

Figure 1. Effects of NGF on axon growth. (A) Average axon length was calculated $0,24,48$ and $72 \mathrm{~h}$ post-NGF treatment. (B) The ratio of axon-possessing versus total cells was calculated. Each column represents mean \pm standard deviation. $\mathrm{n}=3 /$ group. ${ }^{*} \mathrm{P}<0.05$ and ${ }^{* *} \mathrm{P}<0.01$ vs. control. NGF, nerve growth factor; control, untreated cells.
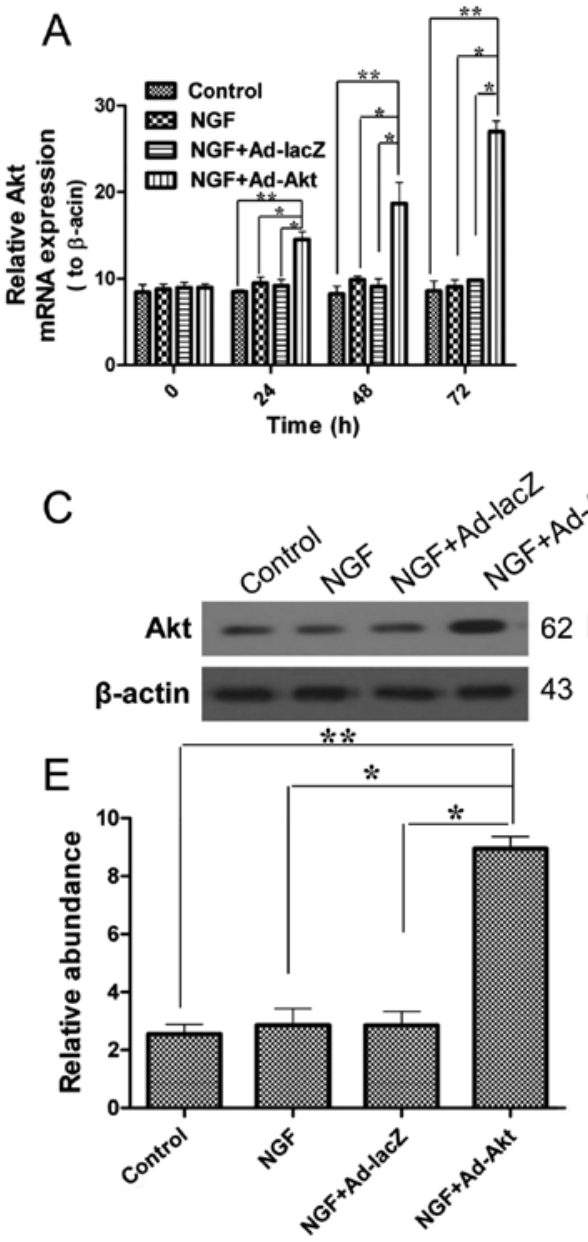
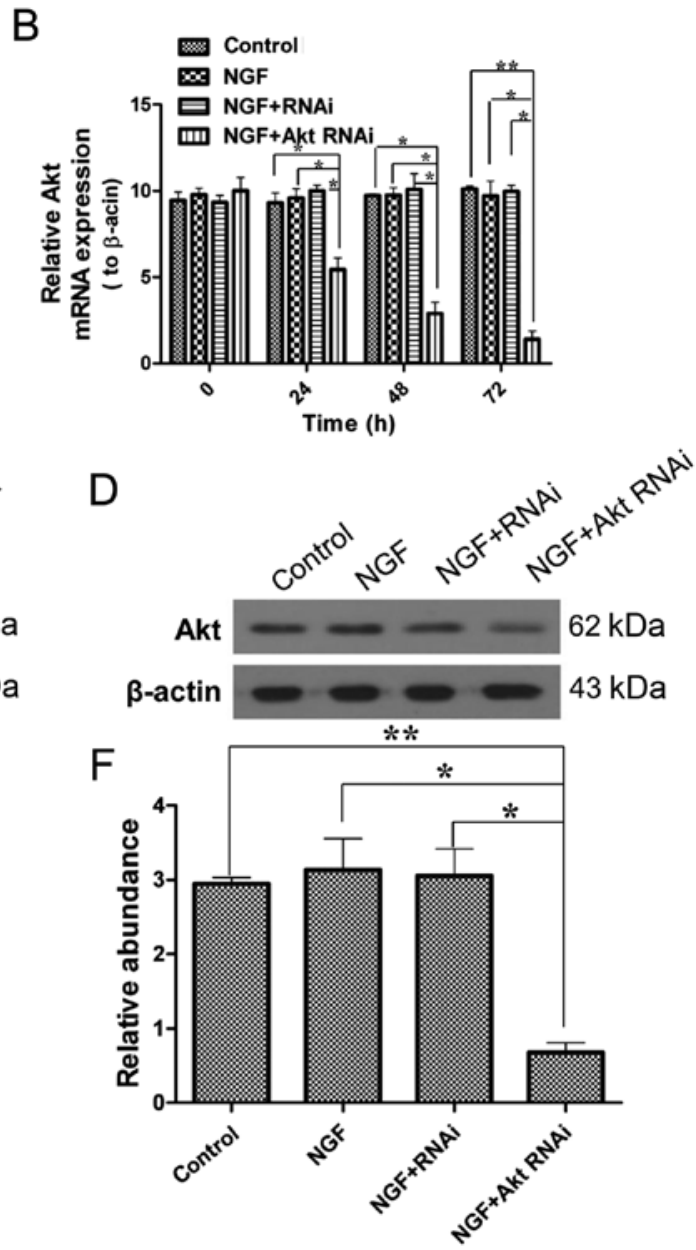

Figure 2. Overexpression and silencing of Akt at the mRNA and protein level. Reverse transcription-quantitative polymerase chain reaction for Akt (A) overexpression and (B) silencing. Western blotting for Akt (C) overexpression and (D) silencing. (E and F) Quantitative analysis of the western blot results. Each column represents mean \pm standard deviation. $\mathrm{n}=3$ /group. ${ }^{*} \mathrm{P}<0.05$ and ${ }^{* *} \mathrm{P}<0.01$ vs. control. NGF, nerve growth factor; control, untreated cells.

increase in the neurites length and also in the number of neurite-possessing cells in a time-dependent manner (Fig. 1). After $72 \mathrm{~h}$ induction, the average axon length was 21.4-fold longer than previously and the percentage of axon-attached neurocytes increased to $62.8 \pm 3.8 \%$. These results demonstrated that NGF could induce neural differentiation of PC12 cells.

Akt is overexpressed or silenced in PC12 cells. Subsequently, in order to conduct the further experiments under defined conditions, the effects of adenovirus transfection and siRNA on PC12 cells were confirmed. Fig. 2 shows that Akt mRNA and protein expression levels were significantly upregulated by adenovirus transfection, and by contrast, siRNA knocked down Akt expression.

Akt promotes the proliferation of PC12 cells. Cell viability is important for neural repair and regeneration, and therefore, PC12 cells were subjected to MTT assays. The results showed 

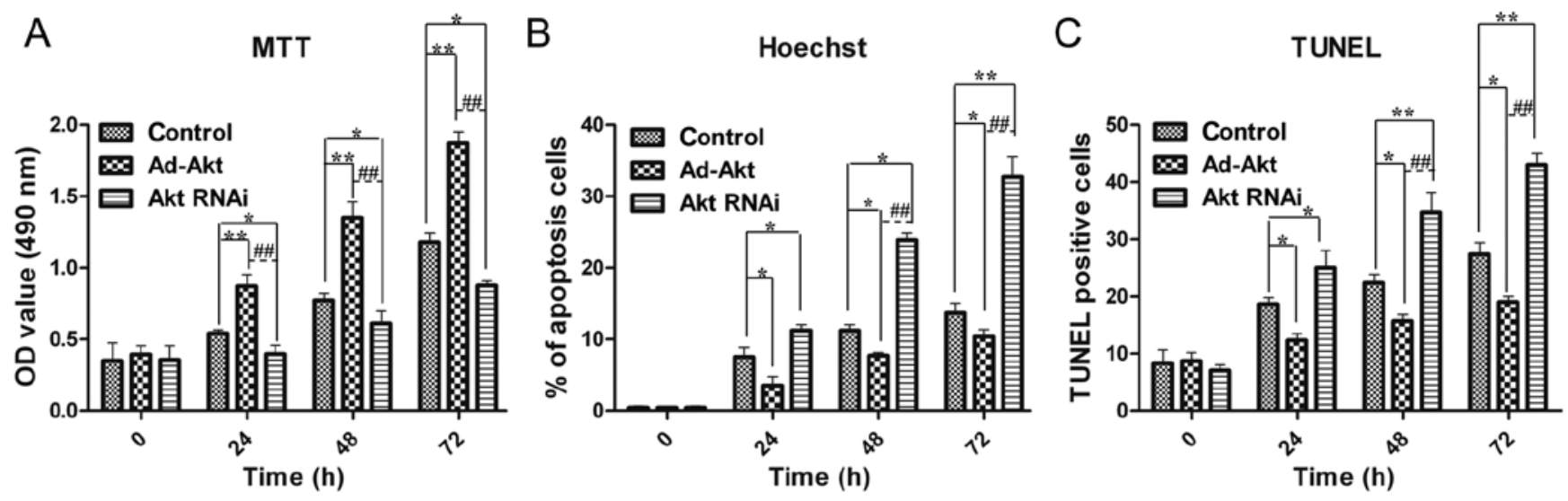

Figure 3. Effects of Akt on proliferation and apoptosis. (A) MTT assay for cell proliferation. OD value was measured at $\gamma=490 \mathrm{~nm}$. (B) Hoechst staining for cell apoptosis. The ratio of apoptotic versus total cells was calculated. (C) TUNEL assay for cell apoptosis. The number of apoptosis-positive cells was counted. Each column represents mean \pm standard deviation. $\mathrm{n}=3$ /group. ${ }^{*} \mathrm{P}<0.05$ and ${ }^{* *} \mathrm{P}<0.01$ vs. control; ${ }^{\# \#} \mathrm{P}<0.01$ Ad Akt vs. Akt RNAi. Control, untreated cells; OD, optical density; TUNEL, terminal deoxynucleotidyltransferase-mediated dUTP nick end labeling.

that cell proliferation was significantly improved in the existence of Akt overexpression, and by contrast, cell growth was inhibited by Akt silencing (Fig. 3A).

Akt inhibits the apoptosis of PC12 cells. As Akt could promote PC12 cells proliferation, Akt was assumed to be able to decrease apoptosis for cell accumulation. The Hoechst and TUNEL experiments were performed to verify this hypothesis. The results proved that Akt overexpression reduced the apoptosis rate to $10.4 \pm 1.0 \%$, while Akt silencing contributed to cell death $(32.7 \pm 2.8 \%$ ) (Fig. 3B). TUNEL results exhibited similar results as the above descriptions (Fig. 3C). These results confirmed the role of Akt in alleviating apoptosis of PC12 cells, which contributed to cell number increase collaborating with cell viability promotion.

Overexpression of Akt enhances axon growth induced by $N G F$. As NGF was proved to promote neural differentiation, the present study aimed to discover a synergetic effector to enhance this function. Overexpression of Akt significantly improved the average axon length $(33.4 \pm 1.6 \mu \mathrm{m})$ compared with the NGF-treated $(26.9 \pm 1.7 \mu \mathrm{m})$ and control groups $(2.14 \pm 0.14 \mu \mathrm{m})$ (Fig. 4A). Furthermore, the number of differentiated cells was also increased by 18.1 and $86.2 \%$ compared with the NGF-treated and control groups, respectively (Fig. 4B).

Silencing of Akt diminishes axon growth induced by NGF. To further identify the function of Akt in axon growth, its expression was knocked out via siRNA. Fig. $4 \mathrm{~A}$ and B showed that $24 \mathrm{~h}$ after siRNA transfection, there was a 1.5 -fold decrease of the average length in the siRNA-treated group, while $48 \mathrm{~h}$ later there was a 1.6-fold decline, compared with the NGF-treated group. Additionally, the differentiated cells were shown to have an average $21 \%$ decrease (from 24 to $48 \mathrm{~h}$ after siRNA transfection). As a result, knockdown of Akt expression attenuated NGF-induced axons outgrowth.

Akt cannot influence axon growth without NGF. The above results confirmed the effects of Akt on the proliferation, apoptosis and axon sprouting of PC12 cells. However, all the aforementioned results were acquired in the presence of NGF, and therefore cannot distinguish whether, how and to what extent Akt alone had participated in these functions. Therefore, the present experiment was conducted to monitor neural differentiation without NGF. The results proved that Akt alone could not promote or attenuate neurite growth, so therefore, Akt did not influence axon growth in the absence of NGF (Fig. 4C and D). This phenomenon confirmed the necessary role of NGF to foster the growth of axons, and Akt could reinforce this effect induced by NGF.

$N G F$ cannot alter the expression of $H O-1, N Q O-1$ and $\gamma-G C S$. As aforementioned, NGF is a necessity for axon extending. Therefore, whether NGF-induced axon growth is associated with the Nrf2/ARE signaling pathway was examined. NGF-treated and non-NGF-treated cells were collected to evaluate the changes of HO-1, NQO- 1 and $\gamma$-GCS expression in mRNA and protein levels. The mRNA expression was not significantly changed in the presence and absence of NGF, which was also similar with the protein expression, and NGF was not closely associated with Nrf2/ARE signaling (Fig. 5).

Akt increases the expression of HO-1, NQO-1 and $\gamma-G C S$ during axon growth. Subsequently, whether Akt has a role in the Nrf2/ARE signaling pathway was investigated. The results showed that Akt overexpression upregulated HO-1, NQO-1 and $\gamma$-GCS expression to 4.3-, 3.2- and 3.8-fold in the mRNA level compared with the control group, respectively (Fig. 6A-C); the western blot results also showed a significant increase in the protein level. By contrast, the knockdown of Akt led to a significant decrease of HO-1, NQO- 1 and $\gamma$-GCS expression at the mRNA and protein levels (Fig. 6D-G). These results confirmed that Akt participates in Nrf2/ARE signaling.

\section{Discussion}

The spine consists of 26 hollow vertebras filled with vast spinal cord containing abundant neurocytes. The axons extending from the neurocytes are responsible for the signal 

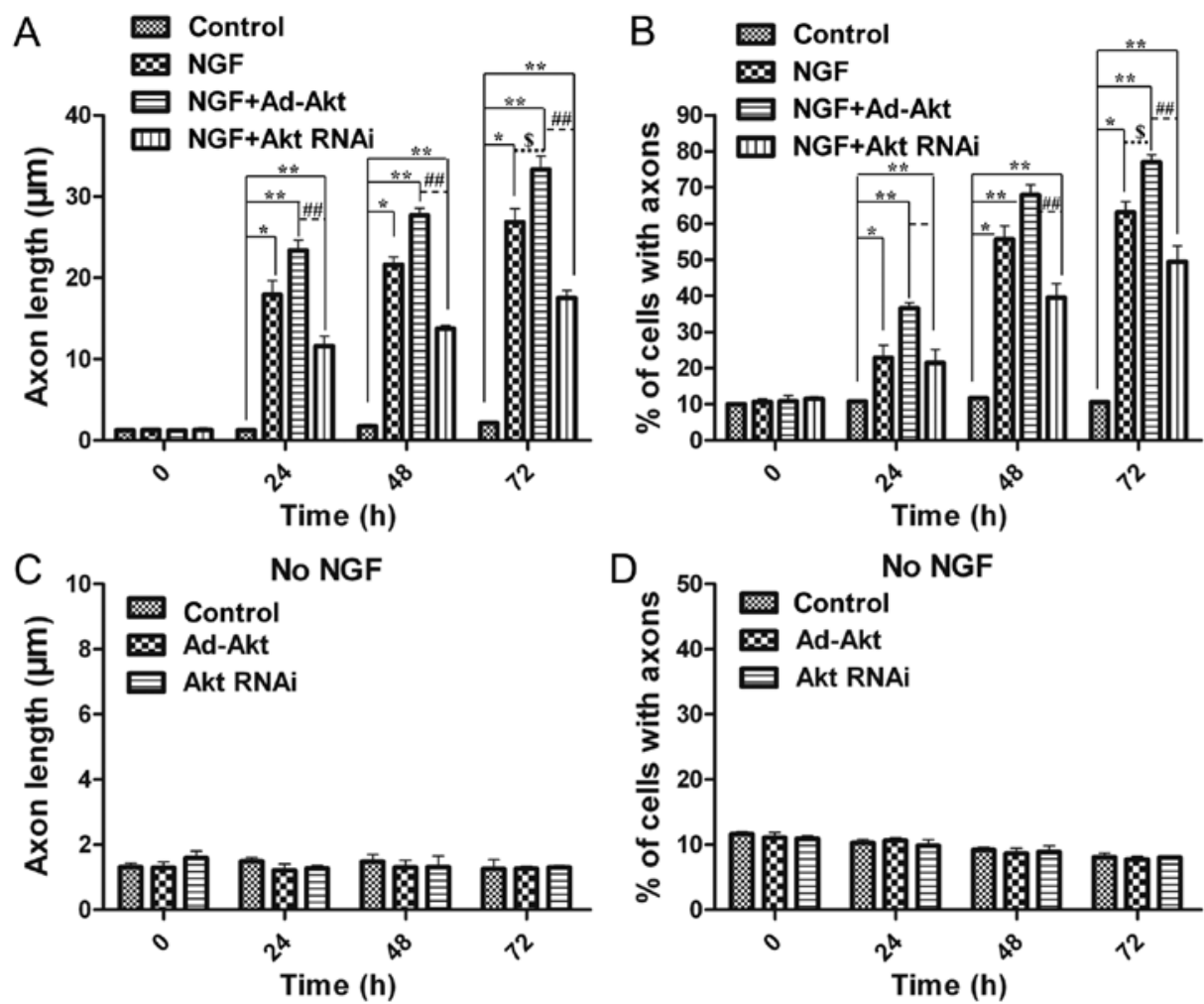

Figure 4. Effects of Akt on axon growth. (A) Average axon length following Akt overexpression and silencing. (B) Data are expressed as a percentage of control. (C and D) Length and percentage of axon following Akt overexpression and silencing without NGF treatment. Each column represents mean \pm standard deviation. $\mathrm{n}=3$ group. ${ }^{*} \mathrm{P}<0.05$ and ${ }^{* *} \mathrm{P}<0.01$ vs. control; ${ }^{* \prime} \mathrm{P}<0.01$ Ad Akt vs. Akt RNAi; ${ }^{\mathrm{P}} \mathrm{P}<0.05$ Ad Akt vs. NGF alone. Control, untreated cells; NGF, nerve growth factor.
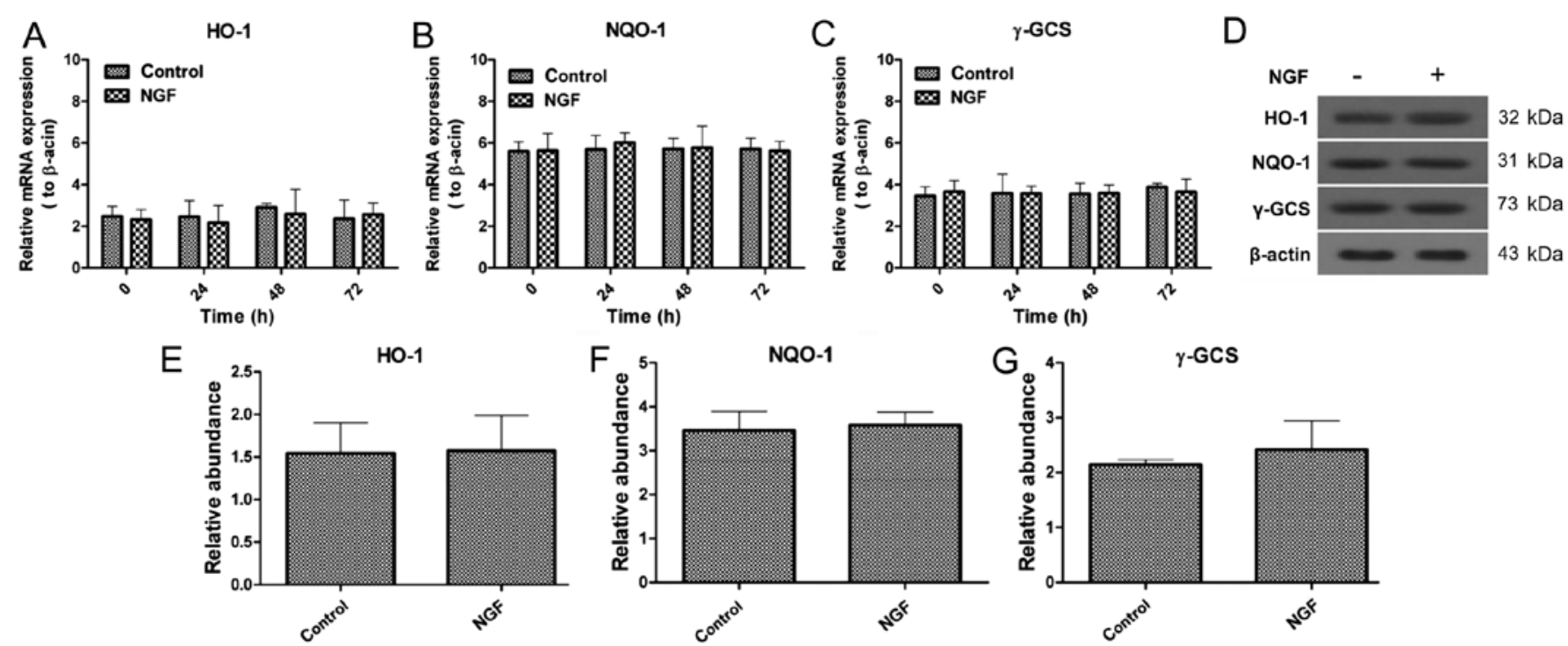

Figure 5. Effects of NGF on HO-1, NQO-1 and $\gamma$-GCS expression. The relative expression was normalized to $\beta$-actin. (A-C) Reverse transcription-quantitative polymerase chain reaction for HO-1, NQO-1 and $\gamma$-GCS mRNA expression. (D) Western blotting for HO-1, NQO-1 and $\gamma$-GCS protein expression. (E-G) Quantitative analysis of the western blot results. Each column represents mean \pm standard deviation. n=3/group. NGF, nerve growth factor; HO-1, heme oxygenase-1; NQO-1, NAD(P)H:quinone oxidoreductase-1; $\gamma$-GCS, $\gamma$-glutamylcysteine synthetase; control, without NGF treatment.

transmission from and to the brain $(25,26)$. SCI is the most serious complication resulting from spinal injury, leading to severe dysfunction and disorder below the injured segment, such as paralysis and quadriplegia. The loss of the function and intercurrent sequelae are mainly due to the death and apoptosis of neurocytes surrounding the lesion sites (27), cutting the signal transmission and losing control of body parts, and the axons could rarely spontaneously regenerate. Therefore, it is important to find techniques for efficient axon regeneration.

Numerous studies have proposed certain methods to promote axon regeneration in animal models. The methods include transplantation of neural stem cell grafts (28); 

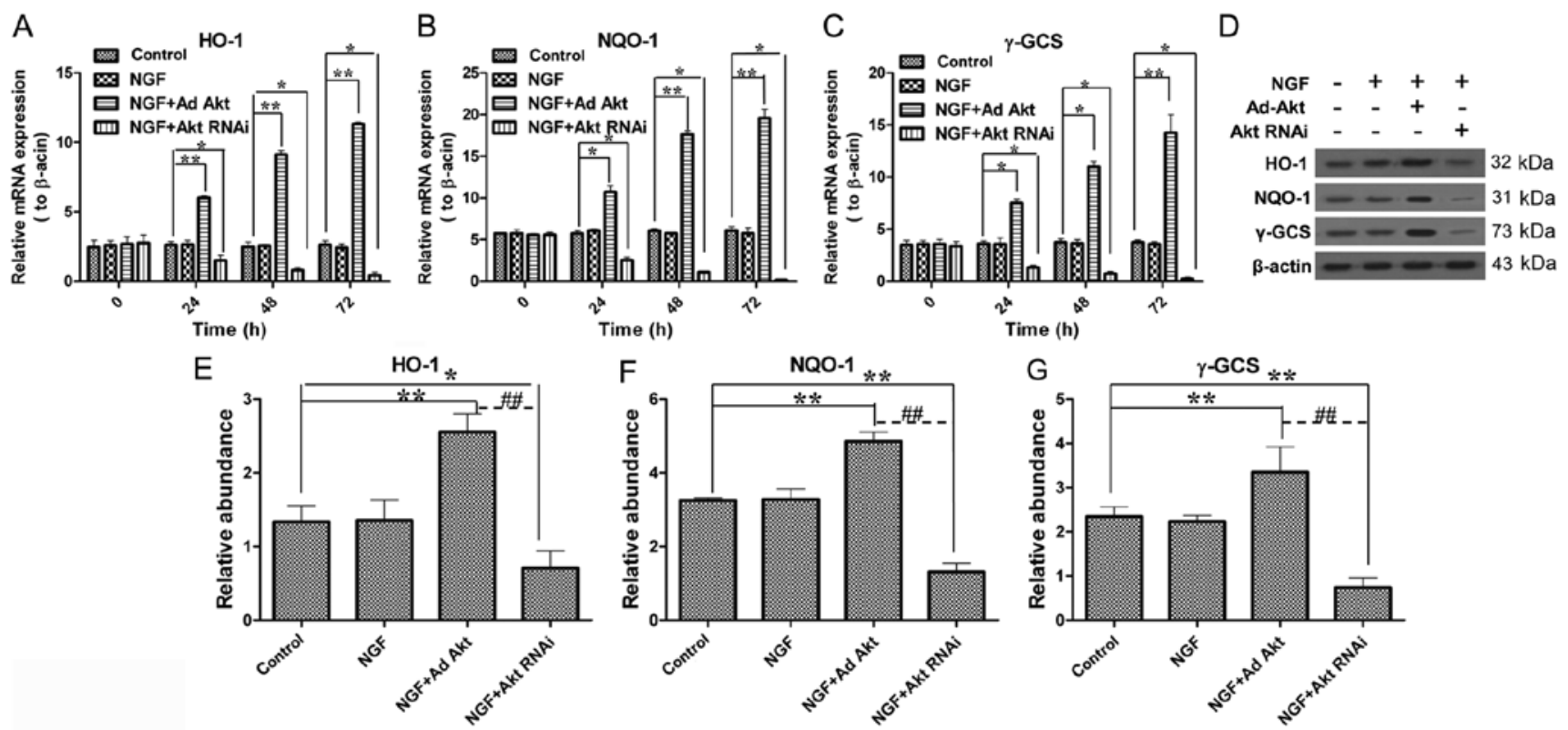

Figure 6. Effects of Akt on HO-1, NQO-1 and $\gamma$-GCS expression. (A-C) mRNA expression of HO-1, NQO-1 and $\gamma$-GCS. (D) Protein expression of HO-1, NQO-1 and $\gamma$-GCS. (E-G) Quantitative analysis of the western blot results. Each column represents mean \pm standard deviation. $n=3 /$ group. ${ }^{*} \mathrm{P}<0.05$ and ${ }^{* *} \mathrm{P}<0.01$ vs. control; ${ }^{\#} \mathrm{P}<0.01$ Ad Akt vs. Akt RNAi. HO-1, heme oxygenase-1; NQO-1, NAD(P)H:quinone oxidoreductase-1; $\gamma$-GCS, $\gamma$-glutamylcysteine synthetase; control, untreated cells; NGF, nerve growth factor.

injection of human induced pluripotent stem cells into the lesion sites (29); inhibition of myelin-associated glycoprotein (MAG) to promote neurites sprouting from transplanted neurons $(30,31)$; avoidance of myelinated tracts $(4,32)$; removal of myelin (33); and the application of antibodies to inhibit myelin inhibitors $(2,34)$. Another means to induce axon regeneration has relied on neurotrophin treatments. For example, different neurotrophin treatments have not only increased neuron regeneration in adult CNS, but also stimulated axonal growth and sprouting following injury $(12,13,35,36)$. All the above methods merely focus on promoting axon regeneration, however, none of the associated signaling mechanisms have been further excavated or investigated.

In the present study, the effects of NGF to promote neural differentiation in PC12 cells were first confirmed. On the basis of NGF, the role of Akt in promoting proliferation and inhibiting apoptosis of PC12 cells was subsequently investigated. Akt was overexpressed and silenced via adenovirus and siRNA transfection. The results showed that increased Akt expression could promote axon growth, contrary to the growth inhibition by Akt silence. Of note, the promoting or inhibitory effects had a precondition that PC12 cells must be treated with NGF. Therefore, NGF is a determinant of axon growth. The Nrf2/ARE signaling pathway is an antioxidative system for neuroprotection. HO-1, NQO- 1 and $\gamma$-GCS are three molecules that have important roles in this antioxidant system. Whether this known neuroprotection is associated with the Akt effects requires elucidating. As expected, Akt overexpression upregulated HO-1, NQO-1 and $\gamma$-GCS expression. By contrast, Akt knockdown had a negative effect on HO-1, NQO-1 and $\gamma$-GCS expression. Therefore, Akt has a positive correlation with Nrf2/ARE signaling. To distinguish the role of NGF in Nrf2/ARE, an experiment was conducted to evaluate HO-1, NQO-1 and $\gamma$-GCS levels with or without
NGF. The results indicated that NGF could not affect Nrf2/ARE signaling.

In the present study, Akt not only promoted the proliferation, but also inhibited the apoptosis of PC12 cells. These all contributed to neuroprotection, such as in SCI treatment, as neurocyte death and reduction are the main reason for SCI complication (37). Additionally, the promotive effect toward axon growth contributed to branching and sprouting of neurocytes to carry signals effectively. However, the present study is limited of animal experiments to verify Akt effects for SCI recovery in vivo, which requires further analysis. In the present study, a synergistic effect was discovered between Akt and NGF. Therefore, only Akt could activate the Nrf2/ARE signaling pathway and its downstream genes. NGF was responsible for neural differentiation, Akt alone had no influence; Akt was able to boost neural differentiation induced by NGF, which was likely to be involved in the Nrf2/ARE pathway. The Nrf2/ARE signaling pathway is a main protective mechanism versus oxidative damage. The upregulation of HO-1, NQO-1 and $\gamma$-GCS by Akt was coordinated with axon growth induced by NGF; they all contribute synergistically and systematically to neuroprotection and functional recovery of neurocytes.

In conclusion, an association between Akt and a potential of the Nrf2/Akt signaling pathway to enhance NGF-induced axon growth was reported, which contributes to the treatment of neural degenerative diseases, such as SCI and its subsequent complications.

\section{Acknowledgements}

The present study was supported by the foundation item of human resource development of the Second Affiliated Hospital of Zhengzhou University. 


\section{References}

1. Schwartz M, Cohen I, Lazarov-Spiegler O, Moalem G and Yoles E: The remedy may lie in ourselves: Prospects for immune cell therapy in central nervous system protection and repair. J Mol Med Berl 77: 713-717, 1999.

2. Schnell L and Schwab ME: Axonal regeneration in the rat spinal cord produced by an antibody against myelin-associated neurite growth inhibitors. Nature 343: 269-272, 1990.

3. Huang DW, McKerracher L, Braun PE and David S: A therapeutic vaccine approach to stimulate axon regeneration in the adult mammalian spinal cord. Neuron 24: 639-647, 1999.

4. Cheng H, Cao Y and Olson L: Spinal cord repair in adult paraplegic rats: Partial restoration of hind limb function. Science 273 510-513, 1996.

5. Howland DR, Bregman BS, Tessler A and Goldberger ME: Transplants enhance locomotion in neonatal kittens whose spinal cords are transected: A behavioral and anatomical study. Exp Neurol 135: 123-145, 1995

6. Rapalino O, Lazarov-Spiegler O, Agranov E, Velan GJ, Yoles E, Fraidakis M, Solomon A, Gepstein R, Katz A, Belkin M, et al: Implantation of stimulated homologous macrophages results in partial recovery of paraplegic rats. Nat Med 4: 814-821, 1998

7. Liu Y, Kim D, Himes BT, Chow SY, Schallert T, Murray M, Tessler A and Fischer I: Transplants of fibroblasts genetically modified to express BDNF promote regeneration of adult rat rubrospinal axons and recovery of forelimb function. J Neurosci 19: 4370-4387, 1999.

8. McDonald JW, Liu XZ, Qu Y, Liu S, Mickey SK, Turetsky D, Gottlieb DI and Choi DW: Transplanted embryonic stem cells survive, differentiate and promote recovery in injured rat spinal cord. Nat Med 5: 1410-1412, 1999.

9. Ramón-Cueto A, Cordero MI, Santos-Benito FF and Avila J: Functional recovery of paraplegic rats and motor axon regeneration in their spinal cords by olfactory ensheathing glia. Neuron 25: 425-435, 2000.

10. Davies SJ, Goucher DR, Doller C and Silver J: Robust regeneration of adult sensory axons in degenerating white matter of the adult rat spinal cord. J Neurosci 19: 5810-5822, 1999.

11. Moon LD, Brecknell JE, Franklin RJ, Dunnett SB and Fawcett JW: Robust regeneration of CNS axons through a track depleted of CNS glia. Exp Neurol 161: 49-66, 2000.

12. Schnell L, Schneider R, Kolbeck R, Barde YA and Schwab ME: Neurotrophin-3 enhances sprouting of corticospinal tract during development and after adult spinal cord lesion. Nature 367: 170-173, 1994

13. Sawai H, Clarke DB, Kittlerova P, Bray GM and Aguayo AJ: Brain-derived neurotrophic factor and neurotrophin-4/5 stimulate growth of axonal branches from regenerating retinal ganglion cells. J Neurosci 16: 3887-3894, 1996.

14. Kobayashi NR, Fan DP, Giehl KM, Bedard AM, Wiegand SJ and Tetzlaff W: BDNF and NT-4/5 prevent atrophy of rat rubrospinal neurons after cervical axotomy, stimulate GAP-43 and Talpha1-tubulin mRNA expression, and promote axonal regeneration. J Neurosci 17: 9583-9595, 1997.

15. Bregman BS, Broude E, McAtee M and Kelley MS: Transplants and neurotrophic factors prevent atrophy of mature CNS neurons after spinal cord injury. Exp Neurol 149: 13-27, 1998.

16. Tischler AS and Greene LA: Nerve growth factor-induced process formation by cultured rat pheochromocytoma cells. Nature 258: 341-342, 1975 .

17. Greene LA and Tischler AS: Establishment of a noradrenergic clonal line of rat adrenal pheochromocytoma cells which respond to nerve growth factor. Proc Natl Acad Sci USA 73: 2424-2428, 1976.
18. Mesner PW, Winters TR and Green SH: Nerve growth factor withdrawal-induced cell death in neuronal PC12 cells resembles that in sympathetic neurons. J Cell Biol 119: 1669-1680, 1992.

19. Ahn JY: Neuroprotection signaling of nuclear akt in neuronal cells. Exp Neurobiol 23: 200-206, 2014.

20. Polivka J Jr and Janku F: Molecular targets for cancer therapy in the PI3K/AKT/mTOR pathway. Pharmacol Ther 142: 164-175, 2014.

21. Kaspar JW, Niture SK and Jaiswal AK: Nrf2:INrf2 (Keap1) signaling in oxidative stress. Free Radic Biol Med 47: 1304-1309, 2009.

22. Ma Q: Role of nrf2 in oxidative stress and toxicity. Annu Rev Pharmacol Toxicol 53: 401-426, 2013.

23. Sarvestani NN, Khodagholi F, Ansari N and Farimani MM: Involvement of p-CREB and phase II detoxifying enzyme system in neuroprotection mediated by the flavonoid calycopterin isolated from Dracocephalum kotschyi. Phytomedicine 20: 939-946, 2013

24. González-Burgos E, Carretero ME and Gómez-Serranillos MP: Nrf2-dependent neuroprotective activity of diterpenoids isolated from Sideritis spp. J Ethnopharmacol 147: 645-652, 2013.

25. Kole MH and Stuart GJ: Signal processing in the axon initial segment. Neuron 73: 235-247, 2012.

26. O'Donnell M, Chance RK and Bashaw GJ: Axon growth and guidance: Receptor regulation and signal transduction. Annu Rev Neurosci 32: 383-412, 2009.

27. McKerracher L: Spinal cord repair: Strategies to promote axon regeneration. Neurobiol Dis 8: 11-18, 2001.

28. Lu P, Kadoya K and Tuszynski MH: Axonal growth and connectivity from neural stem cell grafts in models of spinal cord injury. Curr Opin Neurobiol 27: 103-109, 2014.

29. Lu P, Woodruff G, Wang Y, Graham L, Hunt M, Wu D, Boehle E, Ahmad R, Poplawski G, Brock J, et al: Long-distance axonal growth from human induced pluripotent stem cells after spinal cord injury. Neuron 83: 789-796, 2014.

30. McKeon RJ, Schreiber RC, Rudge JS and Silver J: Reduction of neurite outgrowth in a model of glial scarring following CNS injury is correlated with the expression of inhibitory molecules on reactive astrocytes. J Neurosci 11: 3398-3411, 1991.

31. Davies SJ, Fitch MT, Memberg SP, Hall AK, Raisman G and Silver J: Regeneration of adult axons in white matter tracts of the central nervous system. Nature 390: 680-683, 1997.

32. David S and Aguayo AJ: Axonal elongation into peripheral nervous system 'bridges' after central nervous system injury in adult rats. Science 214: 931-933, 1981

33. Keirstead HS, Hasan SJ, Muir GD and Steeves JD: Suppression of the onset of myelination extends the permissive period for the functional repair of embryonic spinal cord. Proc Natl Acad Sci USA 89: 11664-11668, 1992.

34. Bregman BS, Kunkel-Bagden E, Schnell L, Dai HN, Gao D and Schwab ME: Recovery from spinal cord injury mediated by antibodies to neurite growth inhibitors. Nature 378: 498-501, 1995.

35. Weidner N, Ner A, Salimi N and Tuszynski MH: Spontaneous corticospinal axonal plasticity and functional recovery after adult central nervous system injury. Proc Natl Acad Sci USA 98: 3513-3518, 2001.

36. Coumans JV, Lin TT, Dai HN, MacArthur L, McAtee M, Nash C and Bregman BS: Axonal regeneration and functional recovery after complete spinal cord transection in rats by delayed treatment with transplants and neurotrophins. J Neurosci 21: 9334-9344, 2001.

37. Wang J, Zheng Q, Zhao M and Guo X: Neurocyte apoptosis and expressions of caspase-3 and Fas after spinal cord injury and their implication in rats. J Huazhong Univ Sci Technolog Med Sci 26: 709-712, 2006. 